\title{
Traumatic Brain Injury-Related Deaths by Race/Ethnicity, Sex, Intent, and Mechanism of Injury — United States, 2000-2017
}

\author{
Jill Daugherty, $\mathrm{PhD}^{1}$; Dana Waltzman, $\mathrm{PhD}^{1}$; Kelly Sarmiento, $\mathrm{MPH}^{1}$; Likang Xu, $\mathrm{MD}^{1}$
}

Traumatic brain injury (TBI) affects the lives of millions of Americans each year (1). To describe the trends in TBI-related deaths among different racial/ethnic groups and by sex, CDC analyzed death data from the National Vital Statistics System (NVSS) over an 18-year period (2000-2017). Injuries were also categorized by intent, and unintentional injuries were further categorized by mechanism of injury. In 2017, TBI contributed to 61,131 deaths in the United States, representing $2.2 \%$ of approximately 2.8 million deaths that year. From 2015 to $2017,44 \%$ of TBI-related deaths were categorized as intentional injuries (i.e., homicides or suicides). The leading category of TBI-related death varied over time and by race/ ethnicity. For example, during the last 10 years of the study period, suicide surpassed unintentional motor vehicle crashes as the leading category of TBI-related death. This shift was in part driven by a $32 \%$ increase in TBI-related suicide deaths among non-Hispanic whites. Firearm injury was the underlying mechanism of injury in nearly all (97\%) TBI-related suicides among all groups. An analysis of TBI-related death rates by sex and race/ethnicity found that TBI-related deaths were significantly higher among males and persons who were American Indians/Alaska Natives (AI/ANs) than among all other groups across all years. Other leading categories of TBIrelated deaths included unintentional motor vehicle crashes, unintentional falls, and homicide. Understanding the leading contributors to TBI-related death and identifying groups at increased risk is important in preventing this injury. Broader implementation of evidence-based TBI prevention efforts for the leading categories of injury, such as those aimed at stemming the significant increase in TBI-related deaths from suicide, are warranted.

Data from CDC's NVSS multiple-cause-of-death files were analyzed for 2000-2017. NVSS collects data for all deaths among U.S. residents. TBI-related deaths were classified using codes from the International Classification of Diseases, Tenth Revision (ICD-10) using an established surveillance definition (2). Deaths were classified as TBI-related if any multiple codes for causes of deaths listed in the death record indicated a TBIrelated diagnosis, and the single underlying cause of death was listed as an injury. This methodology represents a change in the calculation of estimates from previous CDC reports $(1,2)$, which did not require that an injury be listed as an underlying

\footnotetext{
*https://www.cdc.gov/nchs/products/databriefs/db328.htm.
}

cause of death. ${ }^{\dagger}$ Data on TBI-related deaths were stratified by year, race/ethnicity, sex, and principal mechanism of injury. Racial/ethnic groups included non-Hispanic white (white), non-Hispanic black (black), non-Hispanic American Indian/ Alaska Native (AI/AN), non-Hispanic Asian/Pacific Islander (Asian/PI), Hispanic, and other. Injuries were categorized first by intent (intentional, unintentional, and undetermined intent). Intentional injuries were further categorized as suicide or homicide. Unintentional injuries were further categorized by mechanism of injury (motor vehicle crashes, falls, being struck by or against an object, or unspecified). Principal mechanism of injury was categorized based on the CDC-recommended external cause of injury mortality matrix for ICD-10 (3) and are presented as the pooled average of 3-year groupings.

Each rate and its corresponding 95\% confidence interval were based on U.S. bridged-race population estimates of the resident population (4). U.S. census population estimates for the year 2000 were used as the standard for age-adjusted rates by direct method (5). T-tests were used to analyze betweengroup differences for rates of TBI-related deaths. Only selected comparisons were tested for statistical significance. Differences with $\mathrm{p}$-values $<0.05$ were considered statistically significant. JoinPoint regression software (version 4.7.0.0; National Cancer Institute) was used to calculate the average annual percent changes of TBI-related death rates from 2000 to 2017 for each race and Hispanic origin group to illustrate trends over time. Average annual percent changes were considered significantly different from zero for p-values $<0.05$. SAS (version 9.4; SAS Institute, Inc.) was used for all statistical analyses.

The overall rate of TBI-related deaths remained constant from 2000 to 2005 , followed by a statistically significant decrease in the overall rate from 2005 to 2010 and then a flattening out from 2010 to 2014. From 2014 to 2017, a small but statistically significant increase in the overall rate of TBI-related deaths occurred (Figure). TBI-related death rates were significantly higher among males of all races than among females throughout the study period $(\mathrm{p}<0.001)$ (Table 1$)$, and age-adjusted rates were significantly higher among AI/AN persons than among other racial/ethnic groups $(\mathrm{p}<0.001)$. From

\footnotetext{
$\dagger$ Previous estimates of TBI-related deaths included all cases in which a TBI-related ICD-10 code was listed in the NVSS mortality record, regardless of whether an injury code was listed as the underlying cause of death. The current methodology only includes deaths for which an injury was more directly related to the cause of death.
} 
FIGURE. Age-adjusted rates* of traumatic brain injury-related deaths, by year and race/ethnicity ${ }^{\dagger}$ — United States, $2000-2017$

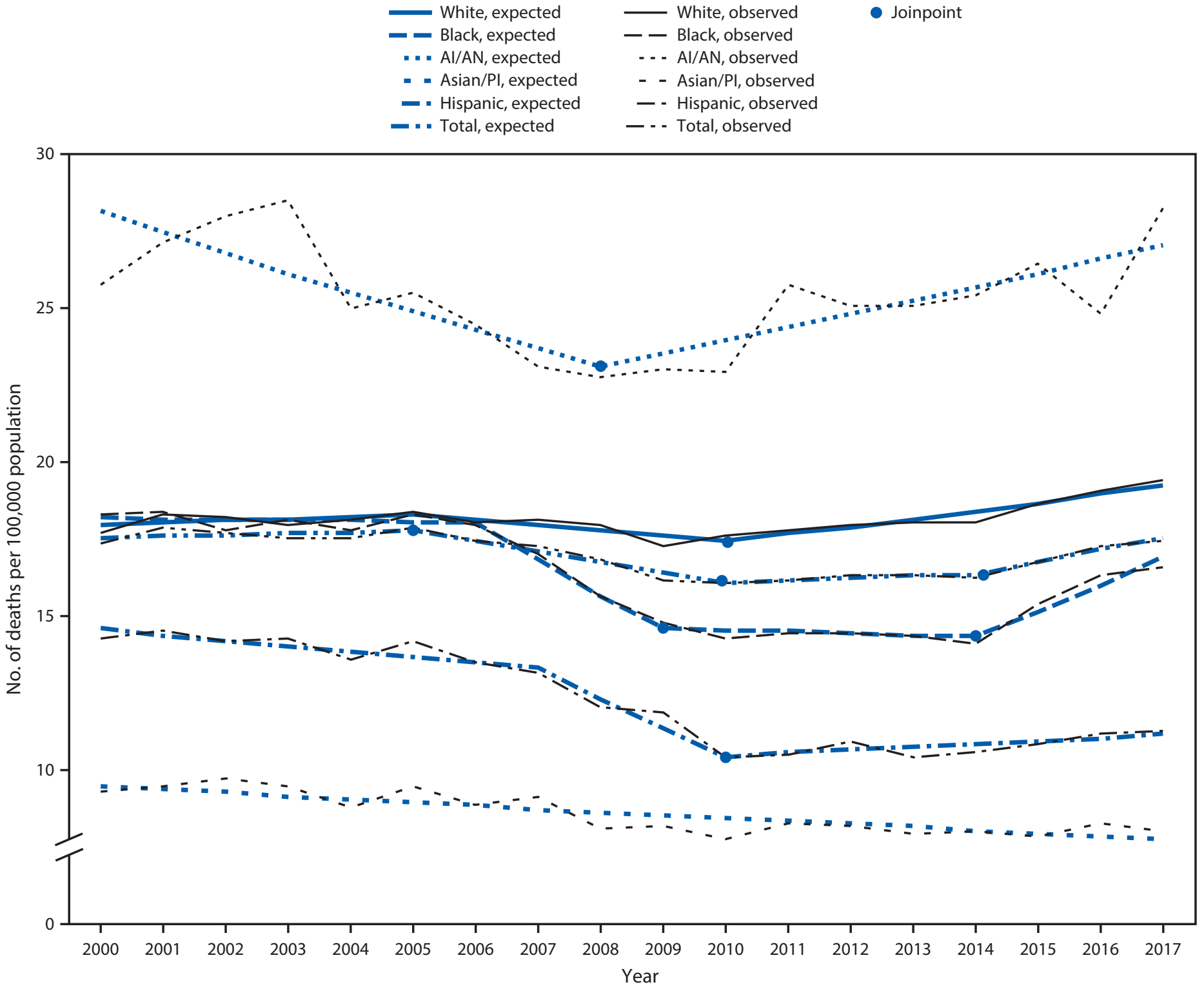

Abbreviations: $\mathrm{Al} / \mathrm{AN}=$ American Indian/Alaska Native; A/PI = Asian/Pacific Islander.

* Per 100,000 population.

† Persons who were white, black, Al/AN, and A/PI were non-Hispanic; Hispanic persons could be of any race.

2001 to 2006, the death rates of whites and blacks were similar ( $>>0.05$ ), but since 2007, the rate of TBI-related deaths has been significantly higher among whites $(\mathrm{p}<0.001)$.

Unintentional TBIs combined across mechanism of injury were responsible for a higher number and rate of deaths than were suicide and homicide across all study years $(\mathrm{p}<0.001)$ (Table 2). Unintentional motor vehicle crashes led to the highest number and rate of all TBI-related deaths from 2000-2002 to 2006-2008 $(\mathrm{p}<0.05)$. Beginning in 2009-2011 and continuing through 2015-2017, suicide was responsible for the most TBI-related deaths $(\mathrm{p}<0.001)$. Across all data years, firearm-related injuries were responsible for approximately $97 \%$ of all TBI-related suicides. The leading category of TBIrelated injury death varied by race/ethnicity and changed for some groups during the study period. For example, from 2000-2002 to 2003-2005, unintentional motor vehicle crashes accounted for the highest rate of TBI-related deaths for whites $(\mathrm{p}<0.001)$. Beginning in 2006-2008 and continuing through 2015-2017, suicide accounted for the highest rate of TBI-related deaths for this group $(\mathrm{p}<0.002)$. Among blacks, homicide was responsible for the highest rate of TBIrelated deaths from 2000-2002 to 2015-2017 ( $\mathrm{p}<0.001)$. 
TABLE 1. Estimated number* and age-adjusted rates ${ }^{\dagger}$ of traumatic brain injury-related deaths, ${ }^{\S}$ by year, sex, and race/ethnicity $\_$United States, 2000-2017**

\begin{tabular}{|c|c|c|c|c|c|c|c|c|c|c|c|c|c|}
\hline \multirow{3}{*}{$\begin{array}{l}\text { Year/ } \\
\text { Sex }\end{array}$} & \multicolumn{11}{|c|}{ Race/Ethnicity } & \multirow{2}{*}{\multicolumn{2}{|c|}{ Total }} \\
\hline & \multicolumn{2}{|r|}{ White } & \multicolumn{2}{|r|}{ Black } & \multicolumn{2}{|c|}{$\begin{array}{c}\text { American Indian/Alaska } \\
\text { Native }\end{array}$} & \multicolumn{2}{|c|}{ Asian/Pacific Islander } & \multicolumn{2}{|r|}{ Hispanic } & \multirow{2}{*}{$\frac{\text { Other }}{\text { No. }}$} & & \\
\hline & No. & Rate $(95 \% \mathrm{Cl})$ & No. & Rate $(95 \% \mathrm{Cl})$ & No. & Rate $(95 \% \mathrm{Cl})$ & No. & Rate $(95 \% \mathrm{Cl})$ & No. & Rate $(95 \% \mathrm{Cl})$ & & No. & Rate $(95 \% \mathrm{Cl})$ \\
\hline \multicolumn{14}{|l|}{2000} \\
\hline Male & 26,497 & $27.6(27.3-28.0)$ & 4,832 & 30.5 & 412 & $38.5(34.3-42.6)$ & 599 & $13.0(11.9-14.2)$ & 3,593 & $22.5(21.6-23.4)$ & 164 & 36,097 & $27.3(27.0-27.6)$ \\
\hline Female & 9,982 & $9.0(8.8-9.2)$ & 1,436 & $8.0(7.5-8.4)$ & 169 & $14.4(12.2-16.7)$ & 291 & $6.0(5.3-6.7)$ & 912 & $6.3(5.8-6.8)$ & 40 & 12,830 & $8.5(8.4-8.7)$ \\
\hline Total & 36,479 & $17.7(17.5-17.9)$ & 6,268 & $18.3(17.8-18.8)$ & 581 & $25.8(23.6-28.1)$ & 890 & $9.3(8.6-9.9)$ & 4,505 & $14.3(13.8-14.8)$ & 204 & 48,927 & $17.4(17.2-17.5)$ \\
\hline \multicolumn{14}{|l|}{2001} \\
\hline Male & , 747 & $28.6(28.3-29.0)$ & 4,915 & $30.8(29.9-31.7)$ & 429 & $39.4(35.2-43.6)$ & 648 & $13.8(12.6-15.0)$ & 3,865 & $22.7(21.8-23.6)$ & 166 & 37,770 & 28.2 \\
\hline Female & 10,307 & $9.1(8.9-9.3)$ & 1,410 & $7.9(7.4-8.3)$ & 184 & $16.0(13.5-18.4)$ & 306 & $5.9(5.2-6.6)$ & 961 & $6.4(6.0-6.9)$ & 43 & 13,211 & $8.7(8.5-8.8)$ \\
\hline Total & 38,054 & $18.3(18.1-18.5)$ & 6,325 & $18.4(17.9-18.8)$ & 613 & $27.2(24.9-29.5)$ & 954 & $9.5(8.8-10.1)$ & 4,826 & $14.5(14.0-15.0)$ & 209 & 50,981 & $17.9(17.7-18.0)$ \\
\hline \multicolumn{14}{|l|}{2002} \\
\hline Male & 27,771 & $28.4(28.1-28.7)$ & 4,811 & $30.0(29.1-30.9)$ & 480 & $42.2(38.1-46.4)$ & 652 & 13.6 (12.4-14.7) & 3,908 & $22.5(21.6-23.4)$ & 186 & 37,808 & $27.9(27.6-28.2)$ \\
\hline Female & 10,400 & $9.1(9.0-9.3)$ & 1,402 & $7.6(7.2-8.0)$ & 171 & $14.7(12.4-17.0)$ & 334 & $6.4(5.7-7.2)$ & 973 & $6.1(5.7-6.5)$ & 31 & 13,311 & $8.6(8.5-8.8)$ \\
\hline Total & 38,171 & $18.2(18.0-18.4)$ & 6,213 & $17.8(17.3-18.2)$ & 651 & $28.0(25.7-30.3)$ & 986 & $9.7(9.1-10.4)$ & 4,881 & $14.2(13.7-14.6)$ & 217 & 51,119 & $17.7(17.6-17.9)$ \\
\hline \multicolumn{14}{|l|}{2003} \\
\hline Male & 27,631 & $28.0(27.7-28.4)$ & 4,923 & 30.0 & 491 & $44.1(39.8-48.4)$ & 671 & $13.3(12.2-14.4)$ & 3,977 & $22.1(21.3-23.0)$ & 119 & 37,812 & $27.6(27.3-27.9)$ \\
\hline Female & 10,439 & $9.0(8.8-9.2)$ & 1,472 & $8.0(7.6-8.4)$ & 162 & $14.2(11.9-16.5)$ & 338 & $6.1(5.4-6.8)$ & 1,038 & $6.4(6.0-6.9)$ & 40 & 13,489 & $8.6(8.5-8.8)$ \\
\hline Total & 38,070 & 18.0 & 6,395 & $18.1(17.7-18.6)$ & 653 & $28.6(26.2-30.9)$ & 1,009 & $9.4(8.8-10.0)$ & 5,015 & $14.2(13.8-14.7)$ & 159 & 51,301 & $17.6(17.4-17.7)$ \\
\hline 2004 & & & & & & & & & & & & & \\
\hline Male & 27,799 & $27.9(27.6-28.3)$ & 4,842 & $29.7(28.8-30.6)$ & 435 & $37.9(34.0-41.8)$ & 604 & $11.7(10.7-12.7)$ & 3,938 & $20.9(20.1-21.7)$ & 117 & 37,735 & $27.2(26.9-27.5)$ \\
\hline Female & 10,921 & $9.4(9.2-9.6)$ & 1,466 & $7.9(7.5-8.3)$ & 163 & $13.2(11.1-15.3)$ & 366 & $6.2(5.6-6.9)$ & 1,012 & $6.1(5.7-6.5)$ & 40 & 13,968 & 8.8( \\
\hline Total & 38,720 & $18.1(18.0-18.3)$ & 6,308 & $17.8(17.4-18.3)$ & 598 & $25.0(22.9-27.1)$ & 970 & $8.7(8.1-9.3)$ & 4,950 & $13.5(13.1-14.0)$ & 157 & 51,703 & 17.5 \\
\hline 2005 & & & & & & & & & & & & & \\
\hline Male & 28,771 & $28.6(28.3-29.0)$ & 5,126 & 30.5 & 471 & 39.0( & 741 & $13.9(12$ & 4,261 & $22.4(2$ & 122 & 39,492 & 28.0 \\
\hline emale & 10,852 & $9.2(9.0-9.4)$ & 1,462 & $7.9(7.5-8.3)$ & 157 & $12.9(10.8-15.0)$ & 352 & $5.8(5.2-6.4)$ & 1,068 & $6.1(5.7-6.5)$ & 25 & 13,916 & $8.6(8$ \\
\hline Total & 39,623 & $18.4(18.2-18.6)$ & 6,588 & $18.3(17.9-18.8)$ & 628 & $25.6(23.5-27.6)$ & 1,093 & $9.5(8.9-10.1)$ & 5,329 & 14.2 & 147 & 53,408 & $17.8(17.7-18.0)$ \\
\hline 2006 & & & & & & & & & & & & & \\
\hline Male & 28,336 & $27.9(27.5-28.2)$ & 5,205 & $30.4(29.5-31.3)$ & 453 & $37.8(34.1-41.5)$ & 703 & $12.6(11.7-13.6)$ & 4,254 & $21.3(20.5-22.1)$ & 105 & 39,056 & $27.3(27.0-27.6)$ \\
\hline Female & 10,905 & $9.2(9$. & 1,401 & $7.4(7.0-7.7)$ & 152 & $12.1(10.1-14.0)$ & 355 & $-6.3)$ & 1,025 & $5.7(5.3$ & 29 & 67 & 3.6) \\
\hline Total & 39,241 & 18.0 & 6,606 & 18.0 & 605 & $24.5(22.4-26.5)$ & 1,058 & $8.9(8.3-9.4)$ & 5,279 & $13.5(13.1-13.9)$ & 134 & 52,923 & $17.4(17.3-17.6)$ \\
\hline 2007 & & & & & & & & & & & & & \\
\hline Male & 28,849 & 28.1 (27. & 4,980 & 28.2 & 422 & 39.2) & 752 & 13.9) & 4,141 & $20.6(1$ & 104 & 39,248 & 27.1 \\
\hline Female & 11,003 & $9.1(8.9-9.2)$ & 1,395 & $7.2(6.8-7.6)$ & 141 & $11.4(9.4-13.3)$ & 373 & $5.8(5.2-6.4)$ & 1,056 & $5.7(5.3-6.1)$ & 29 & 13,997 & $8.4(8.3-8.6)$ \\
\hline Total & 39,852 & $18.1(17.9-18.3)$ & 6,375 & $17.0(16.6-17.4)$ & 563 & $23.1(21.1-25.1)$ & 1,125 & $9.1(8.5-9.6)$ & 5,197 & $13.1(12.7-13.5)$ & 133 & 53,245 & $17.3(17.2-17.5)$ \\
\hline 08 & & & & & & & & & & & & & \\
\hline Iale & 211 & 28.1 & 70 & 26.4 & 43 & & 703 & 11. & 3,810 & 19.0 & 84 & & \\
\hline Female & 0,807 & $8.7(8.6-8.9)$ & 1,253 & $6.4(6.0-6.7)$ & 139 & $9.2-13.0)$ & 327 & $3-5.4)$ & 968 & $5.2(4.8-5.6)$ & 32 & 13,526 & 8.0 \\
\hline Total & 40,018 & $18.0(17.8-18.2)$ & 5,923 & $15.7(15.3-16.1)$ & 569 & $22.7(20.8-24.7)$ & 1,030 & $8.1(7.6-8.6)$ & 4,778 & 12.0 & 116 & 52,434 & $16.8(16.7-17.0)$ \\
\hline 2009 & & & & & & & & & & & & & \\
\hline Male & 28,236 & $26.9(26.6-27.2)$ & 4,346 & $24.5(23.7-25.3)$ & 411 & $33.2(29.8-36.5)$ & 711 & $11.6(10.7-12.5)$ & 3,789 & $18.3(17.6-18.9)$ & 154 & 37,647 & $25.4(25.1-25.6)$ \\
\hline Female & 10,610 & $8.5(8.3-8.6)$ & 1,298 & $6.5(6.1-6.9)$ & 169 & $13.2(11.2-15.3)$ & 362 & $5.2(4.7-5.7)$ & 1,034 & $5.4(5.1-5.8)$ & 43 & 13,516 & $7.9(7.7-8.0)$ \\
\hline Total & 38,846 & $17.2(17.1-17.4)$ & 5,644 & $14.8(14.4-15.2)$ & 580 & $23.0(21.1-25.0)$ & 1,073 & $8.1(7.6-8.6)$ & 4,823 & $11.8(11.4-12.2)$ & 197 & 51,163 & 16.2 \\
\hline $201(x-r)$ & & & & & & & & & & & & & \\
\hline Male & 28,678 & 327.5 (27. & 4,303 & 24.0 & 401 & $3.1)$ & 749 & 11.8 & 3,381 & 16.3( & 144 & 37,656 & 25.3 \\
\hline Female & 10,948 & $8.7(8.5-8.8)$ & 1,168 & $5.8(5.5-6.2)$ & 150 & $12.5(10.4-14.5)$ & 327 & $4.4(3.9-4.9)$ & 975 & $4.9(4.5-5.2)$ & 40 & 13,608 & $7.8(7.6-7.9)$ \\
\hline $\begin{array}{l}\text { Total } \\
2011\end{array}$ & 39,626 & 17.6 (17.4-17.8) & 5,471 & $14.2(13.9-14.6)$ & 551 & $22.9(20.9-24.9)$ & 1,076 & $7.7(7.2-8.2)$ & 4,356 & $10.4(10.0-10.7)$ & 184 & 51,264 & 16.0 \\
\hline Male & ,067 & $27.6(27.3$ & 4,420 & 24.3 & 462 & 1) & 798 & 3.3) & 3,581 & $16.8(1$ & 114 & & 25.4 \\
\hline Female & 11,086 & $8.8(8.6-8.9)$ & 1,237 & $6.0(5.7-6.4)$ & 166 & 13.3 (11.2-15.4) & 384 & $5.1(4.5-5.6)$ & 937 & $4.6(4.3-4.9)$ & 36 & 13,846 & $7.8(7.7-7.9)$ \\
\hline Total & 40,153 & 17.8 (17.6-17.9) & 5,657 & $14.4(14.1-14.8)$ & 628 & $25.7(23.6-27.9)$ & 1,182 & $8.3(7.8-8.8)$ & 4,518 & $10.5(10.1-10.8)$ & 150 & 52,288 & $16.2(16.0-16.3)$ \\
\hline 012 & & & & & & & & & & & & & \\
\hline Male & 29,678 & 3 27.9 (27. & 4,549 & 24.5 & 495 & 4.0) & 797 & 11.7 & 3,700 & 17.3 & 137 & 39 & 25.7 \\
\hline Female & 11,402 & $8.9(8.8-9.1)$ & 1,187 & $5.7(5.4-6.0)$ & 144 & 12.9) & 422 & $5.3(4.8-5.8)$ & 1,045 & $5.0(4.7-5.3)$ & 38 & 14,238 & $7.9(7.8-8.1)$ \\
\hline Total & 41,080 & $18.0(17.8-18.2)$ & 5,736 & $14.4(14.0-14.8)$ & 639 & $25.1(23.1-27.1)$ & 1,219 & $8.1(7.6-8.6)$ & 4,745 & $10.9(10.5-11.2)$ & 175 & 53,594 & $16.3(16.2-16.5)$ \\
\hline 2013 & & & & & & & & & & & & & \\
\hline Male & 30,118 & $328.0(27.7-28.4)$ & 4,525 & $24.0(23.3-24.8)$ & 461 & 38.7 (34.9-42.4) & 841 & $11.5(10.7-12.3)$ & 3,605 & $16.4(15.8-17.0)$ & 120 & 39,670 & $25.5(25.3-25.8)$ \\
\hline Female & 11,588 & $9.0(8.8-9.1)$ & 1,257 & $5.9(5.6-6.3)$ & 160 & $12.7(10.6-14.7)$ & 424 & $5.0(4.5-5.5)$ & 1,044 & $4.8(4.5-5.1)$ & 35 & 14,508 & $7.9(7.8-8.1)$ \\
\hline Total & 41,706 & $18.1(17.9-18.3)$ & 5,782 & $14.4(14.0-14.7)$ & 621 & $25.1(23.1-27.2)$ & 1,265 & $7.9(7.5-8.4)$ & 4,649 & $10.4(10.0-10.7)$ & 155 & 54,178 & $16.3(16.1-16.4)$ \\
\hline
\end{tabular}

See table footnotes on next page. 
Morbidity and Mortality Weekly Report

TABLE 1. (Continued) Estimated number* and age-adjusted rates ${ }^{\dagger}$ of traumatic brain injury-related deaths, ${ }^{\S}$ by year, sex, and race/ ethnicity ? United States, 2000-2017**

\begin{tabular}{|c|c|c|c|c|c|c|c|c|c|c|c|c|c|}
\hline \multirow{3}{*}{$\begin{array}{l}\text { Year/ } \\
\text { Sex }\end{array}$} & \multicolumn{11}{|c|}{ Race/Ethnicity } & \multirow{2}{*}{\multicolumn{2}{|c|}{ Total }} \\
\hline & \multicolumn{2}{|r|}{ White } & \multicolumn{2}{|r|}{ Black } & \multicolumn{2}{|c|}{$\begin{array}{l}\text { American Indian/Alaska } \\
\text { Native } \\
\end{array}$} & \multicolumn{2}{|c|}{ Asian/Pacific Islander } & \multicolumn{2}{|r|}{ Hispanic } & \multirow{2}{*}{$\frac{\text { Other }}{\text { No. }}$} & & \\
\hline & No. & Rate $(95 \% \mathrm{Cl})$ & No. & Rate $(95 \% \mathrm{Cl})$ & No. & Rate $(95 \% \mathrm{Cl})$ & No. & Rate $(95 \% \mathrm{Cl})$ & No. & Rate $(95 \% \mathrm{Cl})$ & & No. & Rate $(95 \% \mathrm{Cl})$ \\
\hline \multicolumn{14}{|l|}{2014} \\
\hline Male & 30,432 & $28.0(27.6-28.3)$ & 4,501 & $23.6(22.8-24.3)$ & 486 & $39.6(35.9-43.4)$ & 888 & $11.6(10.8-12.4)$ & 3,738 & $16.5(15.9-17.1)$ & 152 & 40,197 & 25.5 \\
\hline Female & 11,714 & $9.0(8.8-9.2)$ & 1,242 & $5.9(5.6-6.2)$ & 158 & $12.4(10.4-14.4)$ & 442 & $4.9(4.5-5.4)$ & 1,139 & $5.1(4.8-5.4)$ & 49 & 14,744 & $8.0(7.8-8.1)$ \\
\hline Total & 42,146 & $18.0(17.9-18.2)$ & 5,743 & $14.1(13.7-14.5)$ & 644 & $25.4(23.4-27.5)$ & 1,330 & $8.0(7.5-8.4)$ & 4,877 & $10.6(10.3-10.9)$ & 201 & 54,941 & $16.3(16.1-16.4)$ \\
\hline \multicolumn{14}{|l|}{2015} \\
\hline Male & 31,353 & $28.8(28.5-29.1)$ & 5,007 & $25.8(25.1-26.6)$ & 490 & $39.2(35.5-42.8)$ & 902 & $11.2(10.4-11.9)$ & 3,970 & $16.8(16.2-17.4)$ & 166 & 41,888 & $26.3(26.0-26.5)$ \\
\hline Female & 12,070 & $9.2(9.1-9.4)$ & 1,359 & $6.3(6.0-6.6)$ & 195 & $14.8(12.7-16.9)$ & 478 & $5.0(4.6-5.5)$ & 1,203 & $5.2(4.9-5.5)$ & 53 & 15,358 & $8.2(8.1-8.3)$ \\
\hline Total & 43,423 & $18.6(18.4-18.8)$ & 6,366 & $15.4(15.0-15.8)$ & 685 & $26.5(24.5-28.5)$ & 1,380 & $7.8(7.4-8.2)$ & 5,173 & $10.8(10.5-11.1)$ & 219 & 57,246 & $16.8(16.6-16.9)$ \\
\hline \multicolumn{14}{|l|}{2016} \\
\hline Male & 32,241 & $29.4(29.1-29.8)$ & 5,359 & $27.3(26.5-28.0)$ & 486 & $38.2(34.7-41.8)$ & 988 & $11.6(10.9-12.3)$ & 4,310 & $17.5(16.9-18.1)$ & 141 & 43,525 & $26.9(26.6-27.2)$ \\
\hline Female & 12,501 & $9.5(9.4-9.7)$ & 1,498 & $6.8(6.5-7.2)$ & 166 & $12.4(10.4-14.3)$ & 540 & $5.3(4.9-5.8)$ & 1,275 & $5.2(4.9-5.5)$ & 29 & 16,009 & $8.5(8.3-8.6)$ \\
\hline Total & 44,742 & $19.1(18.9-19.3)$ & 6,857 & $16.4(16.0-16.8)$ & 652 & $24.8(22.9-26.8)$ & 1,528 & $8.2(7.8-8.6)$ & 5,585 & $11.2(10.9-11.5)$ & 170 & 59,534 & $17.3(17.1-17.4)$ \\
\hline \multicolumn{14}{|l|}{2017} \\
\hline Male & 33,209 & 30.0 & 5,577 & $27.8(27.0-28.5)$ & 542 & $42.2(38.5-45.9)$ & 1,041 & $11.9(11.1-12.6)$ & 4,463 & $17.9(17.3-18.4)$ & 129 & 44,961 & $27.4(27.2-27.7)$ \\
\hline Female & 12,688 & $9.6(9.4-9.8)$ & 1,473 & $6.6(6.3-7.0)$ & 210 & $15.5(13.3-17.6)$ & 512 & $4.8(4.4-5.3)$ & 1,254 & $5.1(4.8-5.4)$ & 33 & 16,170 & $8.4(8.3-8.6)$ \\
\hline Total & 45,897 & $19.4(19.2-19.6)$ & 7,050 & $16.6(16.2-17.0)$ & 752 & $28.3(26.2-30.4)$ & 1,553 & $8.0(7.6-8.4)$ & 5,717 & $11.3(10.9-11.6)$ & 162 & 61,131 & $17.5(17.3-17.6)$ \\
\hline
\end{tabular}

Abbreviation: $\mathrm{Cl}=$ confidence interval.

* Death estimates obtained from CDC's National Vital Statistics System. Visits with missing age or sex were excluded; numbers subject to rounding error.

† Per 100,000 population, age-adjusted to the 2000 U.S. standard population, using 12 age groups: $0-4,5-9,10-14,15-19,20-24,25-34,35-44,45-54,55-64,65-74$, $74-84$, and $\geq 85$ years.

$\S$ Record-axis condition codes were used (usually included both part I and part II of entity-axis condition codes). https://www.cdc.gov/nchs/data/datalinkage/ underlying_and_multiple_causes_of_death557_2011.pdf.

9 Persons who were white, black, American Indian/Alaska Native, Asian/ Pacific Islander, or Other were non-Hispanic; Hispanics could be of any race.

** Differences in any two rates were considered statistically significant if their Cls were not overlapping.

Across the study period, the highest rate of TBI-related deaths among AI/AN was attributed to unintentional motor vehicle crashes $(\mathrm{p}<0.05)$. Among Hispanics, unintentional motor vehicle crashes were the most common cause of TBI-related deaths from 2000-2002 to 2006-2008 ( $\mathrm{p}<0.001$ ). During 2009-2011, the rates of TBI-related death from unintentional motor vehicle crashes and unintentional falls were similar ( $\mathrm{p}=0.16$ ) in Hispanics; beginning in 2012-2014 and through 2015-2017, unintentional falls were the most common cause of TBI-related deaths among Hispanics $(\mathrm{p}<0.001)$.

\section{Discussion}

Over the 18-year study period, approximately 960,000 TBI-related deaths occurred in the United States; however, the patterns differed over time and among racial/ethnic groups. Whereas the rates of TBI-related deaths among whites and blacks were similar from 2001 to 2006, the rates among whites subsequently exceeded those among blacks, presumably related to a $32 \%$ increase in TBI-related suicide deaths among whites, from 5.9 per 100,000 during 2006-2008 to 7.8 during 2015-2017. Previous data have documented an increasing prevalence of suicide among whites and AI/ANs (6). These findings suggest that tailored prevention efforts might be needed to help reduce the prevalence of TBI among different groups at risk for injury.
This analysis corroborated findings in a 2017 study of TBIrelated emergency department visits, hospitalizations, and deaths (1) that identified a shift in the leading category of TBIrelated deaths in the United States during the last 10 years from unintentional motor vehicle crashes to suicide. That shift was driven by a significant increase in TBI-related suicide deaths as well as an overall decrease in motor vehicle crash deaths during the last decade ( 7 ). CDC supports suicide prevention efforts by encouraging the use of strategies that reflect the best available evidence, including strengthening access and delivery of suicide care, creating protective environments, teaching coping and problem-solving skills, and identifying and supporting persons at risk (8). Firearm injury was the underlying mechanism of injury in nearly all TBI-related suicides among all groups. Reducing access to lethal means among persons at risk for suicide is an important approach to creating protective environments (8).

Also consistent with previous research, AI/ANs consistently had the highest age-adjusted rates of TBI-related deaths across the study period, and unintentional motor vehicle crashes contributed the highest number and accounted for the highest rate of these TBI-related deaths in all years (9). Lower rates of seat belt use and higher rates of alcohol-related motor vehicle crash deaths among AI/ANs compared with other groups might be contributing factors (9). Expansion of evidence-based strategies 
TABLE 2. Estimated average annual number* and age-adjusted rates ${ }^{\dagger}$ per 100,000 population of traumatic brain injury (TBI)-related deaths $\S$ by year, intent, mechanism of injury, and race/ethnicity ${ }^{\text {I }}$ - United States, 2007-2017**

\begin{tabular}{|c|c|c|c|c|c|c|c|c|c|c|c|c|c|}
\hline \multirow{3}{*}{$\begin{array}{l}\text { 3-year interval/ } \\
\text { mechanism of injury }\end{array}$} & \multicolumn{11}{|c|}{ Race/Ethnicity } & \multirow{2}{*}{\multicolumn{2}{|c|}{ Total }} \\
\hline & \multicolumn{2}{|r|}{ White } & \multicolumn{2}{|r|}{ Black } & \multicolumn{2}{|c|}{$\begin{array}{c}\text { American Indian/Alaska } \\
\text { Native }\end{array}$} & \multicolumn{2}{|c|}{ Asian/Pacific Islander } & \multicolumn{2}{|r|}{ Hispanic } & \multirow{2}{*}{ Other } & & \\
\hline & No. & Rate $(95 \% \mathrm{Cl})$ & No. & Rate $(95 \% \mathrm{Cl})$ & No. & Rate $(95 \% \mathrm{Cl})$ & No. & Rate $(95 \% \mathrm{CI})$ & No. & Rate $(95 \% \mathrm{Cl})$ & & No. & Rate $(95 \% \mathrm{Cl})$ \\
\hline \multicolumn{14}{|l|}{$2000-2002$} \\
\hline $\begin{array}{l}\text { Total unintentional } \\
\text { TBI-related deaths }\end{array}$ & 22,908 & $11.0(10.9-11.1)$ & 2,914 & $8.9(8.7-9.1)$ & 414 & 18.5 (17.4-19.6) & 622 & $6.8(6.5-7.2)$ & 2,940 & $9.3(9.1-9.6)$ & 103 & 29,902 & $10.5(10.4-10.6)$ \\
\hline $\begin{array}{l}\text { Unintentional motor } \\
\text { vehicle crashes }\end{array}$ & 12,416 & $6.3(6.2-6.4)$ & 1,919 & $5.4(5.2-5.5)$ & 311 & $12.8(12.0-13.7)$ & 343 & $3.0(2.8-3.2)$ & 2,014 & $5.3(5.1-5.4)$ & 52 & 17,055 & $6.0(5.9-6.0)$ \\
\hline Unintentional falls ${ }^{\dagger \dagger}$ & 6,496 & $2.8(2.8-2.8)$ & 477 & $1.8(1.7-1.9)$ & 53 & $3.4(2.8-4.0)$ & 194 & $2.8(2.6-3.1)$ & 484 & $2.5(2.4-2.7)$ & 30 & 7,734 & $2.7(2.7-2.8)$ \\
\hline $\begin{array}{l}\text { Unintentionally struck by/ } \\
\text { against an object }\end{array}$ & 304 & $0.1(0.1-0.2)$ & 34 & $0.1(0.1-0.1)$ & $2^{\S \S}$ & $0.1(0.0-0.2)$ & $5^{\S \S}$ & $0.0(0.0-0.1)^{\S \S}$ & 46 & $0.1(0.1-0.2)$ & $2^{\S \S}$ & 393 & $0.1(0.1-0.1)$ \\
\hline $\begin{array}{l}\text { Other unintentional injury, } \\
\text { mechanism } \\
\text { unspecified }\end{array}$ & 3,692 & $1.7(1.7-1.8)$ & 484 & $1.6(1.5-1.7)$ & 48 & $2.2(1.8-2.6)$ & 81 & $0.9(0.8-1.1)$ & 396 & $1.4(1.3-1.5)$ & $19^{\S \S}$ & 4,719 & $1.7(1.6-1.7)$ \\
\hline $\begin{array}{l}\text { Total intentional } \\
\text { TBI-related deaths }\end{array}$ & 14,312 & $6.9(6.9-7.0)$ & 3,258 & $9.0(8.8-9.1)$ & 188 & $7.9(7.3-8.6)$ & 307 & $2.6(2.4-2.7)$ & 1,718 & $4.8(4.6-4.9)$ & 98 & 19,882 & $7.0(6.9-7.0)$ \\
\hline Suicide & 11,909 & $5.7(5.7-5.8)$ & 883 & $2.5(2.4-2.6)$ & 102 & $4.4(3.9-4.9)$ & 164 & $1.4(1.2-1.5)$ & 728 & $2.3(2.2-2.4)$ & 46 & 13,833 & $4.8(4.8-4.9)$ \\
\hline Homicide & 2,403 & $1.2(1.2-1.2)$ & 2,375 & $6.4(6.3-6.6)$ & 86 & $3.6(3.1-4.0)$ & 143 & $1.2(1.1-1.3)$ & 990 & $2.5(2.4-2.6)$ & 51 & 6,049 & $2.1(2.1-2.1)$ \\
\hline $\begin{array}{l}\text { Other (no intent or } \\
\text { mechanism specified)*** }\end{array}$ & 348 & $0.2(0.2-0.2)$ & 96 & $0.3(0.3-0.3)$ & $13^{\S \S}$ & $0.6(0.4-0.8)^{\S \S}$ & $14^{\S \S}$ & $0.1(0.1-0.2)^{\S \S}$ & 79 & $0.2(0.2-0.3)$ & $9^{\S \S}$ & 559 & $0.2(0.2-0.2)$ \\
\hline Total & 37,568 & $18.1(18.0-18.2)$ & 6,269 & $18.2(17.9-18.4)$ & 615 & $27.0(25.7-28.3)$ & 943 & $9.5(9.1-9.9)$ & 4,737 & $14.3(14.1-14.6)$ & 210 & 50,342 & $17.6(17.6-17.7)$ \\
\hline \multicolumn{14}{|l|}{$2003-2005$} \\
\hline $\begin{array}{l}\text { Total unintentional } \\
\text { TBI-related deaths }\end{array}$ & 23,940 & $11.1(11.0-11.2)$ & 3,009 & $9.0(8.8-9.2)$ & 406 & 17.5 (16.4-18.5) & 709 & $6.9(6.6-7.2)$ & 3,181 & $9.3(9.0-9.5)$ & 81 & 31,326 & $10.6(10.5-10.7)$ \\
\hline $\begin{array}{l}\text { Unintentional motor } \\
\text { vehicle crashes }\end{array}$ & 11,827 & $5.9(5.9-6.0)$ & 1,873 & $5.1(5.0-5.3)$ & 276 & $10.8(10.0-11.5)$ & 349 & $2.8(2.6-2.9)$ & 2,146 & $5.1(5.0-5.2)$ & 46 & 16,516 & $5.6(5.6-5.7)$ \\
\hline Unintentional falls ${ }^{\dagger \dagger}$ & 8,325 & $3.4(3.4-3.5)$ & 570 & $2.1(2.0-2.2)$ & 65 & $3.8(3.2-4.4)$ & 272 & $3.3(3.0-3.5)$ & 609 & $3.0(2.8-3.1)$ & 22 & 9,863 & $3.3(3.3-3.4)$ \\
\hline $\begin{array}{l}\text { Unintentionally struck by/ } \\
\text { against an object }\end{array}$ & 286 & $0.1(0.1-0.1)$ & 33 & $0.1(0.1-0.1)$ & $5^{\S \S}$ & $0.2(0.1-0.4)^{\S \S}$ & 7 & $0.1(0.0-0.1)^{\S \S}$ & 50 & $0.1(0.1-0.2)$ & $1^{\S \S}$ & 381 & $0.1(0.1-0.1)$ \\
\hline $\begin{array}{l}\text { Other unintentional injury, } \\
\text { mechanism } \\
\text { unspecified }\end{array}$ & 3,502 & $1.6(1.6-1.7)$ & 534 & $1.7(1.6-1.8)$ & 61 & $2.7(2.3-3.1)$ & 82 & $0.8(0.7-0.9)$ & 376 & $1.1(1.0-1.1)$ & $13^{\S \S}$ & 4,566 & $1.5(1.5-1.6)$ \\
\hline $\begin{array}{l}\text { Total intentional } \\
\text { TBI-related deaths }\end{array}$ & 14,482 & $6.9(6.8-6.9)$ & 3,286 & $8.7(8.5-8.9)$ & 205 & $8.2(7.6-8.9)$ & 302 & $2.2(2.1-2.4)$ & 1,823 & $4.5(4.3-4.6)$ & 69 & 20,168 & $6.8(6.8-6.9)$ \\
\hline Suicide & 12,305 & $5.8(5.7-5.8)$ & 851 & $2.4(2.3-2.4)$ & 112 & $4.6(4.1-5.1)$ & 168 & $1.3(1.1-1.4)$ & 754 & $2.1(2.0-2.1)$ & 36 & 14,225 & $4.8(4.8-4.8)$ \\
\hline Homicide & 2,177 & $1.1(1.1-1.1)$ & 2,436 & $6.3(6.2-6.5)$ & 93 & $3.6(3.2-4.1)$ & 135 & $1.0(0.9-1.1)$ & 1,069 & $2.4(2.3-2.5)$ & 34 & 5,943 & $2.0(2.0-2.0)$ \\
\hline $\begin{array}{l}\text { Other (no intent or } \\
\text { mechanism specified)*** }\end{array}$ & 382 & $0.2(0.2-0.2)$ & 135 & $0.4(0.3-0.4)$ & $15^{\S \S}$ & $0.6(0.5-0.9)^{\S \S}$ & 12 & $0.1(0.1-0.1)^{\S \S}$ & 95 & $0.2(0.2-0.3)$ & $4^{\S \S}$ & 643 & $0.2(0.2-0.2)$ \\
\hline Total & 38,804 & $18.2(18.1-18.3)$ & 6,430 & 18.1 (17.8-18.3) & 626 & $26.3(25.1-27.6)$ & 1,024 & $9.2(8.9-9.6)$ & 5,098 & 14.0 & 154 & 52,137 & $17.6(17.5-17.7)$ \\
\hline \multicolumn{14}{|l|}{$2006-2008$} \\
\hline $\begin{array}{l}\text { Total unintentional } \\
\text { TBI-related deaths }\end{array}$ & 24,156 & $10.8(10.7-10.9)$ & 2,829 & $8.1(7.9-8.2)$ & 372 & $15.4(14.4-16.3)$ & 750 & $6.4(6.2-6.7)$ & 3,133 & $8.4(8.2-8.6)$ & 68 & 31,308 & $10.1(10.1-10.2)$ \\
\hline $\begin{array}{l}\text { Unintentional motor } \\
\text { vehicle crashes }\end{array}$ & 10,662 & $5.3(5.2-5.3)$ & 1,724 & $4.5(4.4-4.6)$ & 243 & $9.2(8.5-9.9)$ & 329 & $2.4(2.3-2.6)$ & 1,952 & $4.2(4.1-4.3)$ & 33 & 14,943 & $4.9(4.9-5.0)$ \\
\hline Unintentional falls ${ }^{\dagger \dagger}$ & 9,920 & $3.9(3.9-3.9)$ & 591 & $2.1(2.0-2.2)$ & 74 & $4.0(3.4-4.5)$ & 345 & $3.4(3.2-3.6)$ & 741 & $3.1(2.9-3.2)$ & 23 & 11,694 & $3.7(3.7-3.8)$ \\
\hline $\begin{array}{l}\text { Unintentionally struck by/ } \\
\text { against an object }\end{array}$ & 283 & $0.1(0.1-0.1)$ & 33 & $0.1(0.1-0.1)$ & $3^{\S \S}$ & $0.1(0.1-0.2)^{\S \S}$ & 7 & $0.1(0.0-0.1)^{\S \S}$ & 55 & $0.1(0.1-0.1)$ & $0^{\S \S}$ & 381 & $0.1(0.1-0.1)$ \\
\hline $\begin{array}{l}\text { Other unintentional injury, } \\
\text { mechanism } \\
\text { unspecified }\end{array}$ & 3,291 & $1.5(1.5-1.5)$ & 481 & $1.4(1.3-1.5)$ & 52 & $2.1(1.8-2.5)$ & 70 & $0.6(0.5-0.7)$ & 385 & $1.0(0.9-1.1)$ & $12^{\S \S}$ & 4,290 & $1.4(1.4-1.4)$ \\
\hline $\begin{array}{l}\text { Total intentional } \\
\text { TBI-related deaths }\end{array}$ & 15,125 & $7.0(7.0-7.1)$ & 3,339 & $8.4(8.3-8.6)$ & 189 & $7.3(6.7-7.9)$ & 301 & $2.1(1.9-2.2)$ & 1,844 & $4.2(4.0-4.3)$ & 55 & 20,854 & $6.8(6.8-6.9)$ \\
\hline Suicide & 12,913 & $5.9(5.9-6.0)$ & 876 & $2.3(2.2-2.4)$ & 107 & $4.2(3.7-4.6)$ & 176 & $1.2(1.1-1.3)$ & 795 & $2.0(1.9-2.1)$ & 36 & 14,903 & $4.8(4.8-4.9)$ \\
\hline Homicide & 2,212 & $1.1(1.1-1.1)$ & 2,463 & $6.1(6.0-6.3)$ & 82 & $3.1(2.7-3.5)$ & 125 & $0.9(0.8-1.0)$ & 1,049 & $2.2(2.1-2.2)$ & $20^{\S \S}$ & 5,950 & $2.0(1.9-2.0)$ \\
\hline $\begin{array}{l}\text { Other (no intent or } \\
\text { mechanism specified) }\end{array}$ & 422 & $0.2(0.2-0.2)$ & 134 & $0.4(0.3-0.4)$ & $18^{\S \S}$ & $0.8(0.6-1.0)^{\S \S}$ & 20 & $0.1(0.1-0.2)^{\S \S}$ & $\S \quad 108$ & $0.3(0.2-0.3)$ & $4^{\S \S}$ & 706 & $0.2(0.2-0.2)$ \\
\hline Total & 39,704 & $18.0(17.9-18.2)$ & 6,301 & $16.9(16.6-17.1)$ & 579 & $23.4(22.3-24.6)$ & 1,071 & $8.7(8.4-9.0)$ & 5,085 & $12.9(12.6-13.1)$ & 128 & 52,867 & $17.2(17.1-17.3)$ \\
\hline
\end{tabular}

See table footnotes on next page.

for reducing the likelihood of injury once a motor vehicle crash has occurred, for example enactment of universal motorcycle helmet laws and enforcement of existing seat belt and child restraint/booster laws, might be beneficial. ${ }^{\$}$

TBI-related homicides disproportionately affected blacks compared with all other groups. CDC's National Center for

\footnotetext{
${ }^{\$}$ https://www.cdc.gov/motorvehiclesafety/index.html.
}

Injury Prevention and Control has created technical packages that outline the best available evidence-based strategies for preventing violence; the strategies are intended to work together and to be used in combination in a multilevel, multisector effort to prevent violence. Implementation might help stop violence before it starts and decrease the rates of TBI-related homicides.

\footnotetext{
https://www.cdc.gov/violenceprevention/pub/technical-packages.html.
} 


\section{Summary}

What is already known about this topic?

Traumatic brain injuries (TBIs) contribute to a substantial number of deaths each year.

What is added by this report?

In 2017, approximately 61,000 TBI-related deaths occurred in the United States. Suicide surpassed motor vehicle crashes as the leading category of TBI-related deaths during 2009-2011 and through 2015-2017. Males and American Indians/Alaska Natives experienced the highest rates of TBI-related death.

What are the implications for public health practice?

Broader implementation of evidence-based prevention strategies for the leading categories of TBI-related death, particularly those aimed at stemming the significant increase in suicide, are warranted. Health care providers can play an important role in assessing patients at increased risk for suicide and providing appropriate interventions.

Falls are the second-leading cause of TBI-related deaths and have been increasing in number and rate, particularly among older adults (1). Health care providers can play an important role in in the prevention of older adult falls. CDC's STEADI** (Stopping Elderly Accidents Deaths and Injuries) initiative can help providers address patient fall risk through the identification of modifiable risk factors and implementation of effective interventions (e.g., strength and balance exercises and medication management).

The findings in this report are subject to at least two limitations. First, misclassification of race and Hispanic origin is a common problem on death certificates, especially for AI/AN, Asian/PI, and Hispanic populations (10). Therefore, for these groups, mortality estimates are most likely underestimates. Second, incomplete reporting or misclassification of cause of death on death certificates might bias estimates of TBI-related deaths.

Understanding the leading contributors to TBI-related death and identifying groups at increased risk is important in preventing this injury. Health care providers can play an important role in assessing patients at increased risk, such as those at risk for suicide, unintentional motor vehicle crashes, or unintentional falls, and provide referrals or tailored interventions.
Corresponding author: Jill Daugherty, jdaugherty@cdc.gov, 404-498-1830.

${ }^{1}$ Division of Injury Prevention, National Center for Injury Prevention and Control, CDC.

All authors have completed and submitted the International Committee of Medical Journal Editors form for disclosure of potential conflicts of interest. No potential conflicts of interest were disclosed.

\section{References}

1. Taylor CA, Bell JM, Breiding MJ, Xu L. Traumatic brain injury-related emergency department visits, hospitalizations, and deaths-United States, 2007 and 2013. MMWR Surveill Summ 2017;66. https://doi. org/10.15585/mmwr.ss6609a1

2. Langlois JA, Rutland-Brown W, Thomas KE. Traumatic brain injury in the United States: emergency department visits, hospitalizations, and deaths. Atlanta, GA: US Department of Health and Human Services, CDC; 2004. https://stacks.cdc.gov/view/cdc/12294

3. CDC. External cause of injury mortality matrix for ICD-10. Atlanta, GA: US Department of Health and Human Services, CDC; 2010. https:// www.cdc.gov/nchs/data/ice/icd10_transcode.pdf

4. National Center for Health Statistics. U.S. Census populations with bridged race categories. Hyattsville, MD: US Department of Health and Human Services, CDC; 2019. https://www.cdc.gov/nchs/nvss/ bridged_race.htm

5. Faul M, Xu L, Wald MM, Coronado VG. Traumatic brain injury in the United States: emergency department visits, hospitalizations, and deaths, 2002-2006. Atlanta, GA: US Department of Health and Human Services, CDC; 2010. https://www.cdc.gov/traumaticbraininjury/pdf/ blue_book.pdf

6. Ivey-Stephenson AZ, Crosby AE, Jack SPD, Haileyesus T, KresnowSedacca MJ. Suicide trends among and within urbanization levels by sex, race/ethnicity, age group, and mechanism of death-United States, 2001-2015. MMWR Surveill Summ 2017;66(No. SS-18). https://doi. org/10.15585/mmwr.ss6618al

7. Hedegaard H. QuickStats: age-adjusted rate of motor vehicle traffic deaths, by urbanization of county of residence-2005 and 2015. MMWR Morb Mortal Wkly Rep 2017;66:567. https://doi. org/10.15585/mmwr.mm6621a6

8. Stone DM, Holland KM, Bartholow BN, Crosby AE, Davis SP, Wilkins N. Preventing suicide: a technical package of policies, programs, and practice. Atlanta, GA: US Department of Health and Human Services, CDC;2017. https://www.cdc.gov/violenceprevention/pdf/ suicideTechnicalPackage.pdf

9. Rutland-Brown W, Wallace LJ, Faul MD, Langlois JA. Traumatic brain injury hospitalizations among American Indians/Alaska Natives. J Head Trauma Rehabil 2005;20:205-14. https://doi. org/10.1097/00001199-200505000-00004

10. Arias E, Heron M, Hakes J; National Center for Health Statistics; US Census Bureau. The validity of race and Hispanic-origin reporting on death certificates in the United States: an update. Vital Health Stat 2 2016; (172):1-21.

\footnotetext{
** https://www.cdc.gov/steadi/index.html.
} 